\title{
Article
}

\section{Complex dynamics of a fractional-order predator-prey interaction with harvesting}

\author{
Rizwan Ahmed \\ Department of Mathematics, National College of Business Administration and Economics, Rahim Yar Khan, Pakistan.; \\ rizwanahmed488@gmail.com
}

Received: 31 July 2020; Accepted: 10 September 2020; Published: 19 October 2020.

\begin{abstract}
Harvesting has a strong impact on the dynamic evolution of a population subjected to it. In this paper, a fractional-order predator-prey interaction is studied with harvesting affecting both predator and prey populations. Local stability of the coexistence equilibrium point is discussed depending upon the harvesting of prey. Moreover, period-doubling and Neimark-Sacker bifurcations are studied for a wide range of constant harvesting effort of prey.
\end{abstract}

Keywords: Predator-prey interaction, stability, Neimark-Sacker bifurcation, period doubling bifurcation.

MSC: 37N25, 39A28, 39A30, 92D25.

\section{Introduction}

$\mathbf{F}$ ractional differential equations are more suitable for modeling many real-life phenomena than ordinary differential equations. Fractional calculus has seen significant development over the past few decades. Chaos is a very interesting nonlinear phenomenon which has been intensively studied due to its useful applications in science and technology [1-3]. Studying chaos in fractional-order dynamical systems is an interesting topic as well. The discrete fractional equations recently attracted researchers in the modeling of the biological populations and interaction of the species [4-7]. Harvesting has a strong impact on the dynamic evolution of a population subjected to it. The effects of predator harvesting were reported in [8,9], harvesting among two fish species and its effects were studied in [10].

In this paper, the predator-prey model is considered with harvesting effort affecting both species directly. We studied the following fractional-order predator-prey model.

$$
\left\{\begin{array}{l}
\frac{d^{\alpha} x(t)}{d t^{\alpha}}=a x(t)(1-x(t))-b x(t) y(t)-c x(t), \\
\frac{d^{\alpha} y(t)}{d t^{\alpha}}=-d y(t)+e x(t) y(t)-f y(t) .
\end{array}\right.
$$

By using discretization of fractional order using piecewise constant arguments methods $[11,12]$ to (1) fractional model we have the following discrete model

$$
\left\{\begin{array}{l}
x(t+1)=x(t)+\frac{k^{\alpha}}{\Gamma(\alpha+1)}[a x(t)(1-x(t))-b x(t) y(t)-c x(t)], \\
y(t+1)=y(t)+\frac{k^{\alpha}}{\Gamma(\alpha+1)}[-d y(t)+e x(t) y(t)-f y(t)] .
\end{array}\right.
$$

where $0<\alpha \leq 1$ is the fractional-order and $k$ is the step size of discretization, $x(t)$ is the population of prey at time $t, y(t)$ is the population of predator at time $t, a$ is the rate of growth of prey, $b$ is the interaction rate, $c$ is the constant harvesting effort of prey, $d$ is the mortality rate of predator, $e$ is the conversion rate of prey, $f$ is the constant harvesting effort of predator. All parameters are assumed to be positive.

The paper is organized as follows. In Section 2, we investigate the topological classification of the unique positive equilibrium point of the system (2). In Section 3, we discuss period-doubling bifurcation and in Section 4 , we discuss Neimark-Sacker bifurcation at positive steady-state. In Section 5, some numerical examples are presented to verify our theoretical results. In Section 6, some concluding remarks are presented. 


\section{Local stability of positive equilibrium point}

The system (2) has three equilibrium points.

$$
E_{0}(0,0), E_{1}\left(\frac{a-c}{a}, 0\right), E_{2}\left(\frac{d+f}{e}, \frac{a e-a d-a f-c e}{b e}\right) .
$$

Note that if $a e-a d-a f-c e>0$, then $E_{2}$ is the unique positive equilibrium point of (2). $E_{0}$ is the mutual extinction, $E_{1}$ is the predator extinction and $E_{3}$ is coexistence equilibrium point of system (2). For bilogically meaningful we concentrated on coexistence equilibrium point $E_{2}$. The jacobian matrix of (2) evaluated at $E_{2}$ is given by

$$
J\left(E_{2}\right)=\left[\begin{array}{cc}
1-\frac{a M(d+f)}{e} & \frac{-b M(d+f)}{e} \\
-\frac{(c e+a(d-e+f)) M}{b} & 1
\end{array}\right],
$$

where $M=\frac{k^{\alpha}}{\Gamma(\alpha+1)}$. The corresponding characteristic polynomial is

$$
F(\lambda)=\lambda^{2}-\left(2-\frac{a M(d+f)}{e}\right) \lambda+1+a(d+f) M^{2}-c M^{2}(d+f)-\frac{a M(d+f)(1+M(d+f))}{e} .
$$

By simple computations we have

$$
\begin{aligned}
F(0) & =1+M^{2}(d+f)(a-c)-\frac{a M(d+f)(1+M(d+f))}{e}, \\
F(1) & =\frac{M^{2}(d+f)(a e-a d-a f-c e)}{e} \\
F(-1) & =4+M^{2}(d+f)(a-c)-\frac{a M(d+f)(2+M(d+f))}{e} .
\end{aligned}
$$

Since $a e-a d-a f-c e>0$, therefore $F(1)>0$. For stability analysis we use the following result.

Lemma 1. [13]: Let $F(\lambda)=\lambda^{2}-A \lambda+B$, where $A, B$ are constants. Suppose $F(1)>0$ and $\lambda_{1}, \lambda_{2}$ are roots of $F(\lambda)=0$, then
(i) $\left|\lambda_{1}\right|<1$ and $\left|\lambda_{2}\right|<1$ iff $F(-1)>0$ and $F(0)<1$.
(ii) $\left|\lambda_{1}\right|<1$ and $\left|\lambda_{2}\right|>1$ iff $F(-1)<0$.
(iii) $\left|\lambda_{1}\right|>1$ and $\left|\lambda_{2}\right|>1$ iff $F(-1)>0$ and $F(0)>1$.
(iv) $\lambda_{1}=-1$ and $\left|\lambda_{2}\right| \neq 1$ iff $F(-1)=0$ and $F(0) \neq \pm 1$.
(v) $\lambda_{1}$ and $\lambda_{2}$ are complex and $\left|\lambda_{1}\right|=\left|\lambda_{2}\right|=1$ iff $A^{2}-4 B<0$ and $F(0)=1$.

Theorem 1. If $(a, b, c, d, e, f, M) \in \mathbb{R}_{+}^{7}, a e-a d-a f-c e>0$ and $\lambda_{1}, \lambda_{2}$ are the roots of $F(\lambda)=0$ given by (3), then (i) $\left|\lambda_{1}\right|<1$ and $\left|\lambda_{2}\right|<1$ iff

$$
a-\frac{a(1+d M+f M)}{e M}<c<a+\frac{4}{(d+f) M^{2}}-\frac{a(2+d M+f M)}{e M},
$$

(ii) $\left|\lambda_{1}\right|<1$ and $\left|\lambda_{2}\right|>1$ iff

$$
c>a+\frac{4}{(d+f) M^{2}}-\frac{a(2+d M+f M)}{e M},
$$

(iii) $\left|\lambda_{1}\right|>1$ and $\left|\lambda_{2}\right|>1$ iff

$$
c<\min \left\{a-\frac{a(1+d M+f M)}{e M}, a+\frac{4}{(d+f) M^{2}}-\frac{a(2+d M+f M)}{e M}\right\},
$$

(iv) $\lambda_{1}=-1$ and $\left|\lambda_{2}\right| \neq 1$ iff

$$
c=a+\frac{4}{(d+f) M^{2}}-\frac{a(2+d M+f M)}{e M}, c \neq a-\frac{a(1+d M+f M)}{e M}, c \neq a+\frac{2}{(d+f) M^{2}}-\frac{a(1+d M+f M)}{e M},
$$

(v) $\lambda_{1}$ and $\lambda_{2}$ are complex and $\left|\lambda_{1}\right|=\left|\lambda_{2}\right|=1$ iff 


$$
c=a-\frac{a(1+d M+f M)}{e M}, \frac{a(d+f) M}{e}<4
$$

The following corollary is an immediate consequence of Theorem 1.

Corollary 1. If $(a, b, c, d, e, f, M) \in \mathbb{R}_{+}^{7}$, ae - ad-af-ce $>0$, then the unique positive equilibrium point $E_{2}$ of (2) is:

(i) a sink and therefore locally asymptotically stable if

$$
a-\frac{a(1+d M+f M)}{e M}<c<a+\frac{4}{(d+f) M^{2}}-\frac{a(2+d M+f M)}{e M},
$$

(ii) saddle point and therefore unstable if

$$
c>a+\frac{4}{(d+f) M^{2}}-\frac{a(2+d M+f M)}{e M},
$$

(iii) source or repeller and therefore unstable if

$$
c<\min \left\{a-\frac{a(1+d M+f M)}{e M}, a+\frac{4}{(d+f) M^{2}}-\frac{a(2+d M+f M)}{e M}\right\},
$$

and

(iv) non-hyperbolic if

$$
c=a+\frac{4}{(d+f) M^{2}}-\frac{a(2+d M+f M)}{e M}, c \neq a-\frac{a(1+d M+f M)}{e M}, c \neq a+\frac{2}{(d+f) M^{2}}-\frac{a(1+d M+f M)}{e M},
$$

or

$$
c=a-\frac{a(1+d M+f M)}{e M}, \frac{a(d+f) M}{e}<4 .
$$

\section{Period doubling bifurcation}

In this section, we investigate that system (2) undergoes period doubling bifurcation $[14,15]$ at the positive equilibrium point $E_{2}$. Consider

$$
\begin{aligned}
\Lambda= & \left\{(a, b, c, d, e, f, M) \in \mathbb{R}_{+}^{7} \mid a e-a d-a f-c e>0, c=a+\frac{4}{(d+f) M^{2}}-\frac{a(2+d M+f M)}{e M}\right. \\
& \left.c \neq a-\frac{a(1+d M+f M)}{e M}, c \neq a+\frac{2}{(d+f) M^{2}}-\frac{a(1+d M+f M)}{e M}\right\} .
\end{aligned}
$$

We discuss the period doubling bifurcation of the system (2) at $E_{2}$ when parameters vary in a small neighborhood of $\Lambda$. Taking $c$ as bifurcation parameter, we consider a perturbation of the system (2) as follows:

$$
\left\{\begin{array}{l}
x(t+1)=x(t)+M[a x(t)(1-x(t))-b x(t) y(t)-(c+\delta) x(t)] \\
y(t+1)=y(t)+M[-d y(t)+e x(t) y(t)-f y(t)]
\end{array}\right.
$$

where $M=\frac{k^{\alpha}}{\Gamma(\alpha+1)}$ and $|\delta| \ll 1$ is small perturbation parameter.

We define a transformation by $\xi(t)=x(t)-\frac{d+f}{e}$ and $\eta(t)=y(t)-\frac{a(e-d-f)-(c+\delta) e}{b e}$ to transfer the equilibrium point $E_{2}$ to $(0,0)$. Under this transformation system (4) will become

$$
\left[\begin{array}{l}
\xi(t+1) \\
\eta(t+1)
\end{array}\right]=\left[\begin{array}{cc}
a_{11} & a_{12} \\
a_{21} & 1
\end{array}\right]\left[\begin{array}{l}
\xi(t) \\
\eta(t)
\end{array}\right]+\left[\begin{array}{l}
a_{1} \xi^{2}(t)+a_{2} \xi(t) \eta(t) \\
b_{1} \xi(t) \delta+b_{2} \xi(t) \eta(t)
\end{array}\right],
$$

where $a_{11}=1-\frac{a(d+f) M}{e}, a_{12}=-\frac{b M(d+f)}{e}, a_{21}=\frac{2(a M(d+f)-2 e)}{b M(d+f)}, a_{1}=-a M, a_{2}=-b M, b_{1}=-\frac{e M}{b}, b_{2}=e M$. Eigenvalues of linearized part of (5) are -1 and $\lambda=3-\frac{a M(d+f)}{e}$. We construct an invertible matrix $T$ as 
$T=\left[\begin{array}{cc}\frac{b M(d+f)}{2 e-a M(d+f)} & -\frac{b M(d+f)}{2 e} \\ 1 & 1\end{array}\right]$. Now we apply the transformation $\left[\begin{array}{l}\xi(t) \\ \eta(t)\end{array}\right]=T\left[\begin{array}{l}u(t) \\ v(t)\end{array}\right]$, to the system (5) and we get

$$
\left[\begin{array}{c}
u(t+1) \\
v(t+1)
\end{array}\right]=\left[\begin{array}{cc}
-1 & 0 \\
0 & \lambda
\end{array}\right]\left[\begin{array}{l}
u(t) \\
v(t)
\end{array}\right]+\left[\begin{array}{l}
F(u(t), v(t), \delta) \\
G(u(t), v(t), \delta)
\end{array}\right]
$$

where

$$
\begin{aligned}
& F(u(t), v(t), \delta)=a_{1} u^{2}(t)+a_{2} v^{2}(t)+a_{3} u(t) v(t)+a_{4} u(t) \delta+a_{5} v(t) \delta, \\
& G(u(t), v(t), \delta)=b_{1} u^{2}(t)+b_{2} v^{2}(t)+b_{3} u(t) v(t)+b_{4} u(t) \delta+b_{5} v(t) \delta
\end{aligned}
$$

with

$$
\begin{aligned}
& a_{1}=\frac{b e M\left(-a M^{2}(d+f)^{2}+2 e(-2+d M+f M)\right)}{(2 e-a(d+f) M)(4 e-a(d+f) M)}, \\
& a_{2}=\frac{b M(-2 e+a(d+f) M)(a(d+f) M+e(-2+d M+f M))}{2 e(4 e-a(d+f) M)}, \\
& a_{3}=\frac{a b(d+f) M^{2}(2+d M+f M)}{8 e-2 a(d+f) M}, \\
& a_{4}=\frac{e(d+f) M^{2}}{a M(d+f)-4 e^{\prime}} \\
& a_{5}=-\frac{(d+f) M^{2}(-2 e+a(d+f) M)}{8 e-2 a(d+f) M}, \\
& b_{1}=\frac{2 b e^{2} M(2+d M+f M)}{(2 e-a(d+f) M)(4 e-a(d+f) M)^{\prime}}, \\
& b_{2}=-\frac{b M\left(-4 a e(d+f) M+a^{2}(d+f)^{2} M^{2}+2 e^{2}(2+d M+f M)\right)}{2 e(4 e-a(d+f) M)}, \\
& b_{3}=\frac{a b(d+f) M^{2}(a(d+f) M+e(-2+d M+f M))}{(2 e-a(d+f) M)(4 e-a(d+f) M)}, \\
& b_{4}=-\frac{2 e^{2} M^{2}(d+f)}{(2 e-a(d+f) M)(4 e-a(d+f) M)^{\prime}}, \\
& b_{5}=\frac{e(d+f) M^{2}}{4 e-a(d+f) M} .
\end{aligned}
$$

Next we determine the center manifold $W^{c}(0,0)$ of the system $(6)$ at the equilibrium point $(0,0)$ in a small neighbourhood of $\delta=0$. By using center manifold theory [16-18], we know that there exist a center manifold $W^{c}(0,0)$, which can be approximately represented as follows:

$$
W^{c}(0,0)=\left\{(u(t), v(t)) \in \mathbb{R}^{2} \mid v(t)=W(u(t), \delta)=m_{1} u^{2}(t)+m_{2} \delta u(t)+m_{3} \delta^{2}+O\left((|u(t)|+|\delta|)^{3}\right)\right\} .
$$

By using first equation of system (6), we have

$$
v(t+1)=W(u(t+1), \delta)=-m_{2} \delta u(t)+m_{1} u^{2}(t)+m_{3} \delta^{2}+O\left((|u(t)|+|\delta|)^{3}\right) .
$$

By using second equation of system (6), we have

$$
v(t+1)=\lambda v(t)+G(u(t), v(t), \delta)=\left(b_{4}+\lambda m_{2}\right) \delta u(t)+\left(b_{1}+\lambda m_{1}\right) u^{2}(t)+\lambda m_{3} \delta^{2}+O\left((|u(t)|+|\delta|)^{3}\right) .
$$

Comparing the coefficients for (7) and (8), we have

$$
m_{1}=\frac{b_{1}}{1-\lambda}, m_{2}=-\frac{b_{4}}{1+\lambda}, m_{3}=0 .
$$


Then the center manifold can be approximated as follows

$$
v(t)=\frac{b_{1}}{1-\lambda} u^{2}(t)-\frac{b_{4}}{1+\lambda} \delta u(t)+O\left((|u(t)|+|\delta|)^{3}\right) .
$$

Substituting (9) in the first equation of the system (6), we have

$$
\begin{aligned}
u(t+1)= & -u(t)+a_{1} u^{2}(t)+a_{4} u(t) \delta+\frac{a_{3} b_{1}}{1-\lambda} u^{3}(t)+\left(\frac{a_{5} b_{1}}{1-\lambda}-\frac{a_{3} b_{4}}{1+\lambda}\right) u^{2}(t) \delta \\
& -\frac{a_{5} b_{4}}{1+\lambda} u(t) \delta^{2}+O\left((|u(t)|+|\delta|)^{3}\right)=\tilde{F}(u(t), \delta) .
\end{aligned}
$$

For period doubling bifurcation we need the following two quantities

$$
L=\left(\frac{\partial^{2} \tilde{F}}{\partial u(t) \partial \delta}+\frac{1}{2} \frac{\partial \tilde{F}}{\partial \delta} \frac{\partial^{2} \tilde{F}}{\partial u^{2}(t)}\right)_{u(t)=0, \delta=0}
$$

and

$$
M=\left(\frac{1}{6} \frac{\partial^{3} \tilde{F}}{\partial u^{3}(t)}+\left(\frac{1}{2} \frac{\partial^{2} \tilde{F}}{\partial u^{2}(t)}\right)^{2}\right)_{u(t)=0, \delta=0}
$$

By simple computations, we have

$$
L=a_{4}, M=a_{1}^{2}+\frac{a_{3} b_{1}}{1-\lambda}
$$

By using above calculations we have the following result for period doubling bifurcation of the system (2).

Theorem 2. If $(a, b, c, d, e, f, M) \in \Lambda$ and $L \neq 0, M \neq 0$ then the system (2) goes under period doubling bifurcation at the equilibrium point $E_{2}$. Moreover, if $M>0$ (or $M<0$ ) then period-2 orbits that bifurcate from the equilibrium point $E_{2}$ are stable (or unstable).

\section{Neimark-Sacker bifurcation}

In this section, we investigate that system (2) undergoes Neimark-Sacker bifurcation $[19,20]$ at the positive equilibrium point $E_{2}$. Consider

$$
\Omega=\left\{(a, b, c, d, e, f, M) \in \mathbb{R}_{+}^{7} \mid a e-a d-a f-c e>0, c=a-\frac{a(1+d M+f M)}{e M}, \frac{a(d+f) M}{e}<4\right\}
$$

where $M=\frac{k^{\alpha}}{\Gamma(\alpha+1)}$.

We discuss the Neimark-Sacker bifurcation of the system (2) at $E_{2}$ when parameters vary in a small neighbourhood of $\Omega$. Taking $c$ as bifurcation parameter, we consider a perturbation of the system (2) as follows:

$$
\left\{\begin{array}{l}
x(t+1)=x(t)+M[a x(t)(1-x(t))-b x(t) y(t)-(c+\delta) x(t)] \\
y(t+1)=y(t)+M[-d y(t)+e x(t) y(t)-f y(t)]
\end{array}\right.
$$

where $M=\frac{k^{\alpha}}{\Gamma(\alpha+1)}$ and $|\delta| \ll 1$ is small perturbation parameter.

We define a transformation by $\xi(t)=x(t)-\frac{d+f}{e}$ and $\eta(t)=y(t)-\frac{a(e-d-f)-(c+\delta) e}{b e}$ to transfer the equilibrium point $E_{2}$ to $(0,0)$. Under this transformation system (10) will become

$$
\left[\begin{array}{l}
\xi(t+1) \\
\eta(t+1)
\end{array}\right]=\left[\begin{array}{cc}
a_{11} & a_{12} \\
a_{21} & 1
\end{array}\right]\left[\begin{array}{l}
\xi(t) \\
\eta(t)
\end{array}\right]+\left[\begin{array}{c}
-b M \xi(t) \eta(t)-a M \xi^{2}(t) \\
e M \xi(t) \eta(t)
\end{array}\right]
$$

where $a_{11}=1-\frac{a M(d+f)}{e}, a_{12}=-\frac{b M(d+f)}{e}, a_{21}=\frac{a-e M \delta}{b}$. 
The characteristic equation of the linearized part of the system $(11)$ at equilibrium point $(0,0)$ is

$$
\lambda^{2}-p(\delta) \lambda+q(\delta)=0
$$

where $p(\delta)=2-\frac{a M(d+f)}{e}$, and $q(\delta)=1-d M^{2} \delta-f M^{2} \delta$.

The roots of (12) are complex with $\left|\lambda_{1,2}\right|=1$ which are given by $\lambda_{1,2}=\frac{p(\delta) \pm i \sqrt{4 q(\delta)-p^{2}(\delta)}}{2}$. By computations, we obtain $\left|\lambda_{1}\right|=\left|\lambda_{2}\right|=\sqrt{q(\delta)}$, and $\left(\frac{d\left|\lambda_{1}\right|}{d \delta}\right)_{\delta=0}=\left(\frac{d\left|\lambda_{2}\right|}{d \delta}\right)_{\delta=0}=-\frac{(d+f) M^{2}}{2}<0$.

By simple computations, we can check that $p(0)=2-\frac{a(d+f) M}{e}$. Since $\frac{a(d+f) M}{e}<4$, therefore we have $-2<p(0)<2$. If we set $p(0)=0$, then we obtain $\frac{a(d+f) M}{e}=2$ and if we set $p(0)=1$, then we obtain $\frac{a(d+f) M}{e}=1$. Since $\lambda_{1}^{m}, \lambda_{2}^{m} \neq 1$ for $m=1,2,3,4$ at $\delta=0$ is equivalent to $p(0) \neq \pm 2,0,1$, therefore $\lambda_{1}^{m}, \lambda_{2}^{m} \neq 1$ for $m=1,2,3,4$ at $\delta=0$ if $(a, b, c, d, e, f, M) \in \Omega$ and following conditions are satisfied:

$$
\frac{a(d+f) M}{e} \neq 1,2
$$

Now we use the following transformation to convert the linear part of (11) into its canonical form at $\delta=0$.

$$
\left[\begin{array}{l}
\xi(t) \\
\eta(t)
\end{array}\right]=\left[\begin{array}{cc}
-\frac{b(d+f) M}{e} & 0 \\
\frac{a M(d+f)}{2 e} & -\frac{1}{2} \sqrt{4-\left(-2+\frac{a M(d+f)}{e}\right)^{2}}
\end{array}\right]\left[\begin{array}{c}
u(t) \\
v(t)
\end{array}\right]
$$

Under the transformation (14), the system (11) will become

$$
\left[\begin{array}{l}
u(t+1) \\
v(t+1)
\end{array}\right]=\left[\begin{array}{cc}
\alpha & -\beta \\
\beta & \alpha
\end{array}\right]\left[\begin{array}{l}
u(t) \\
v(t)
\end{array}\right]+\left[\begin{array}{l}
f(u(t), v(t)) \\
g(u(t), v(t))
\end{array}\right]
$$

where

$$
\begin{aligned}
\alpha & =1-\frac{a M(d+f)}{2 e}, \\
\beta & =\frac{1}{2} \sqrt{4-\left(-2+\frac{a M(d+f)}{e}\right)^{2}} \\
f(u, v) & =\frac{a b M^{2}(d+f)}{2 e} u^{2}+\frac{b M}{2} \sqrt{4-\left(-2+\frac{a M(d+f)}{e}\right)^{2}} u v, \\
g(u, v) & =\frac{a b M^{3}(a+2 e)(d+f)^{2}}{2 e \sqrt{a M(d+f)(4 e-a M(d+f))}} u^{2}+\frac{b(a-2 e)(d+f) M^{2}}{2 e} u v .
\end{aligned}
$$

We define the following real number $L$, which analyses the direction in which the closed invariant curve occurs in a system undergoing Neimark-Sacker bifurcation method:

$$
L=\left(\left[-\operatorname{Re}\left(\frac{\left(1-2 \lambda_{1}\right) \lambda_{2}^{2}}{1-\lambda_{1}} \eta_{20} \eta_{11}\right)-\frac{1}{2}\left|\eta_{11}\right|^{2}-\left|\eta_{02}\right|^{2}+\operatorname{Re}\left(\lambda_{2} \eta_{21}\right)\right]\right)_{\delta=0},
$$

where

$$
\begin{aligned}
\eta_{20} & =\frac{1}{8}\left[f_{u u}-f_{v v}+2 g_{u v}+i\left(g_{u u}-g_{v v}-2 f_{u v}\right)\right] \\
\eta_{11} & =\frac{1}{4}\left[f_{u u}+f_{v v}+i\left(g_{u u}+g_{v v}\right)\right] \\
\eta_{02} & =\frac{1}{8}\left[f_{u u}-f_{v v}-2 g_{u v}+i\left(g_{u u}-g_{v v}+2 f_{u v}\right)\right] \\
\eta_{21} & =\frac{1}{16}\left[f_{u u u}+f_{u v v}+f_{u u v}+g_{v v v}+i\left(g_{u u u}+g_{u v v}-f_{u u v}-f_{v v v}\right)\right] .
\end{aligned}
$$


From calculations the computed partial derivatives are

$$
\begin{aligned}
f_{u u} & =\frac{a b M^{2}(d+f)}{e}, \\
f_{u v} & =\frac{b M}{2} \sqrt{4-\left(-2+\frac{a M(d+f)}{e}\right)^{2},} \\
f_{v v} & =f_{u u u}=f_{v v v}=f_{u v v}=f_{u u v}=0, \\
g_{v v} & =g_{u u u}=g_{v v v}=g_{u v v}=g_{u u v}=0, \\
g_{u u} & =\frac{a b M^{3}(a+2 e)(d+f)^{2}}{e \sqrt{a M(d+f)(4 e-a M(d+f))}}, \\
g_{u v} & =\frac{b M^{2}(d+f)(a-2 e)}{2 e} .
\end{aligned}
$$

Due to above calculations, we have the following result for existence and direction of Neimark-Sacker bifurcation.

Theorem 3. Assume that $(a, b, c, d, e, f, M) \in \Omega$ and conditions (13) are satisfied. If $L \neq 0$, then the system (2) goes under Neimark-Sacker bifurcation at the unique positive equilibrium point $E_{2}$ when the parameter $c$ varies in a small neighbourhood of $c=a-\frac{a(1+d M+f M)}{e M}$. Furthermore, if $L<0$, then an attracting invariant closed curve bifurcates from the equilibrium point for $c>a-\frac{a(1+d M+f M)}{e M}$, and if $L>0$, then a repelling invariant closed curve bifurcates from the equilibrium point for $c<a-\frac{a(1+d M+f M)}{e M}$.

\section{Numerical examples}

In this section some interesting numerical examples are provided to validate our theoretical discussions on various qualitative aspects of the model.

Example 1. We select the parameters values as $a=200, b=0.8, d=2.3, e=40, f=0.5, k=0.15, \alpha=0.95$ and initial conditions $x(0)=0.1, y(0)=8$. For these values the unique positive equilibrium point of (2) is $(0.07,11.2316)$. The eigenvalues of $J\left(E_{2}\right)$ for these values are $\lambda_{1}=-1, \lambda_{2}=0.643641$, which confirms that the system (2) undergoes period doubling bifurcation at $(0.07,11.2316)$ as bifurcation parameter passes through $c=177.015$. We plot bifurcation diagrams for both prey and predator populations for $c \in[176,180]$. (see Figure 1)
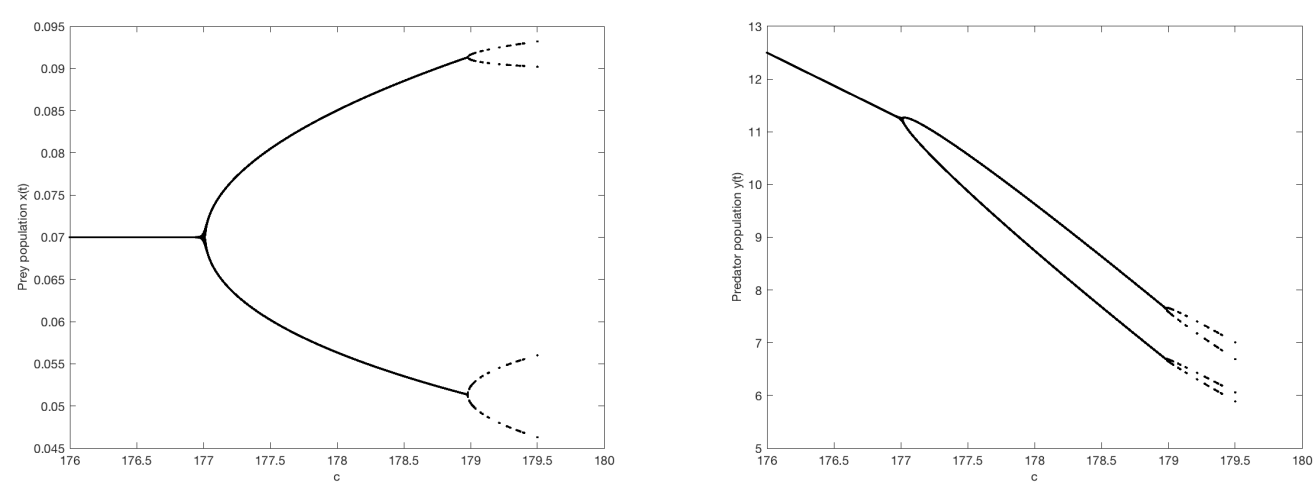

Figure 1. Bifurcation diagrams for prey and predator populations with $a=200, b=0.8, d=2.3, e=40, f=$ $0.5, k=0.15, \alpha=0.95, x(0)=0.1, y(0)=8, c \in[176,180]$.

Example 2. We select the parameters values as $a=200, b=0.8, d=2.3, e=40, f=0.5, k=0.15, \alpha=0.95$ and initial conditions $x(0)=0.1, y(0)=35$. For these values the unique positive equilibrium point of (2) is $(0.07,37.1336)$. The eigenvalues of $J\left(E_{2}\right)$ for these values are $\lambda_{1}=-0.17818+0.983998 i, \lambda_{2}=-0.17818-$ 
$0.983998 i$ with $\left|\lambda_{1,2}\right|=1$, which confirms that the system (2) undergoes Neimark-Sacker bifurcation at $(0.07,37.1336)$ as bifurcation parameter passes through $c=156.293$. We plot bifurcation diagrams for both prey and predator populations for $c \in[156,156.7]$. (see Figure 2 )
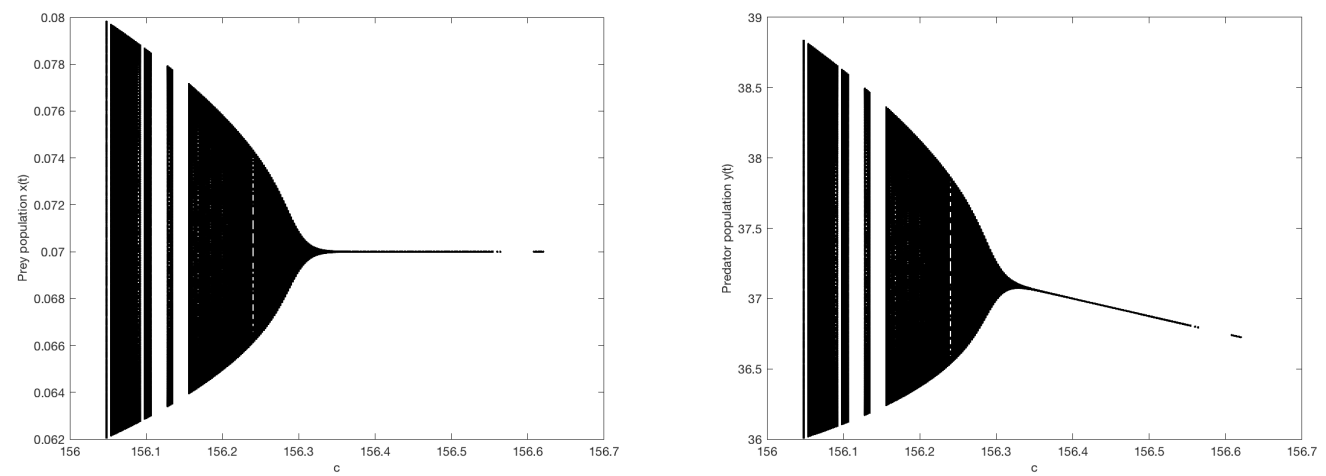

Figure 2. Bifurcation diagrams for prey and predator populations with $a=200, b=0.8, d=2.3, e=40, f=$ $0.5, k=0.15, \alpha=0.95, x(0)=0.1, y(0)=35, c \in[156,156.7]$.

Example 3. We select the parameters values as $a=20, b=10, d=2, e=40, f=1, k=0.11, \alpha=0.9$ and the initial conditions $x(0)=0.073, y(0)=0.35$. The positive equilibrium point for these values is $(0.075,0.35058)$. For these values the equilibrium point is locally asymptotically stable iff $14.9942<c<77.0381$. We plot phase portraits for $c=14,14.9942,15,15.2$. Clearly one can see that for $c=14$, the equilibrium point $(0.35058,0.142621)$ is unstable, whereas for $c=15$ and $c=15.2$ it is stable. For $c=14.9942$, the system undergoes bifurcation. (See figure (3))
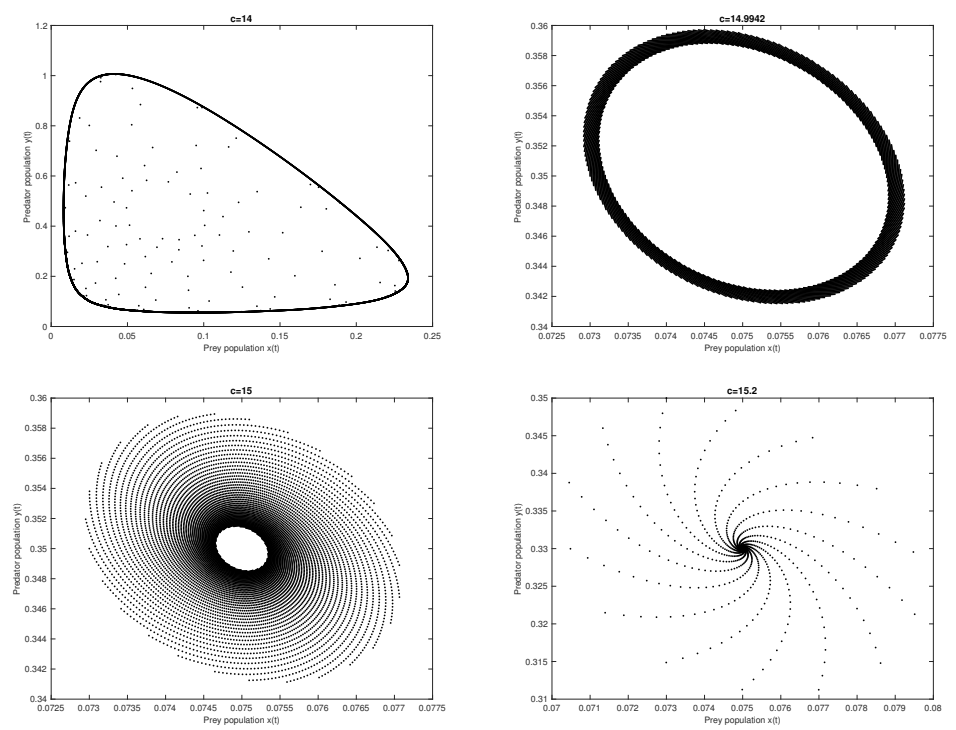

Figure 3. Phase portraits for prey and predator populations with $a=20, b=10, d=2, e=40, f=1, k=$ $0.11, \alpha=0.9, x(0)=0.05, y(0)=0.3$ for $c=14,14.9942,15,15.2$

\section{Conclusion}

We studied a fractional-order predator-prey model with harvesting in both species. We study the existence and local stability of coexistence equilibrium point $E_{2}$ of system (2) and their dependence in the form of constant harvesting effort of prey. Moreover, the system (2) goes under period-doubling bifurcation 
and Neimark-Sacker bifurcation under certain conditions on constant harvesting effort of prey. Numerical examples are presented to support our theoretical results.

Conflicts of Interest: "The author declares no conflict of interest."

\section{References}

[1] He, Z., \& Lai, X. (2011). Bifurcation and chaotic behavior of a discrete-time predator-prey system. Nonlinear analysis real world applications, 12(1), 403-417.

[2] Singh, H., Dhar, J., \& Bhatti, H. S. (2015). Discrete-time bifurcation behavior of a prey-predator system with generalized predator. Advances in Difference Equations, 2015(1), 206.

[3] Din, Q. (2018). Stability, bifurcation analysis and chaos control for a predator-prey system. Journal of Vibration and Control, 25(3), 612-626.

[4] Elsadany, A. A., \& Matouk, A. E. (2015). Dynamical behaviors of fractional-order Lotka Volterra predator-prey model and its discretization. Applied Mathematics and Computation, 49, 269-283.

[5] Nosrati, K., Shafiee, M. (2017). Dynamic analysis of fractional-order singular Holling type-II predator-prey system. Applied Mathematics and Computation, 313, 159-179.

[6] Wang, Z., Xie, Y., Lu, J., \& Li, Y. (2019). Stability and bifurcation of a delayed generalized fractional-order prey-predator model with interspecific competition. Applied Mathematics and Computation, 347, 360-369.

[7] Alidousti, J., \& Ghafari, E. (2020). Dynamic behavior of a fractional order prey-predator model with group defense. Chaos, Solitons \& Fractals, 134, 109688.

[8] Myerscough, M. R., Gray, B. F., Hogarth, W. L., \& Norbury, J. (1992). An analysis of an ordinary differential equation model for a two-species predator-prey system with harvesting and stocking. Journal of Mathematical Biology, 30, 389-411.

[9] Hu, D., Cao, H. (2017). Stability and bifurcation analysis in a predator-prey system with Michaelis-Menten type predator harvesting. Nonlinear Analysis Real World Applications, 33, 58-82.

[10] Kar, T. K., \& Chaudhuri, K. S. (2003). On non-selective harvesting of two competing fish species in the presence of toxicity. Ecological Modelling, 161(1), 125-137.

[11] Agarwal, R. P., El-Sayed, A. M., \& Salman, S. M. (2013). Fractional-order ChuaŠs system: discretization, bifurcation and chaos. Advances in Difference Equations, 2013(1), 1-13.

[12] Zhang, J., Nan, J., Du, W., Chu, Y., \& Luo, H. (2016). Dynamic analysis for a fractional-order autonomous chaotic system. Discrete Dynamics in Nature and Society, 2016, 8712496.

[13] Liu, X. L., \& Xiao D. M. (2007). Complex dynamic behaviors of a discrete-time predator-prey system. Chaos, Solitons E Fractals, 32(1), 80-94.

[14] Agiza, H. N., Elabbasy, E. M., EL-Metwally, H., \& Elsadany, A. A. (2009). Chaotic dynamics of a discrete prey-predator model with Holling type II. Nonlinear Analysis Real World Applications, 10(1), 116-129.

[15] Jana, D. (2013). Chaotic dynamics of a discrete predator-prey system with prey refuge. Applied Mathematics and Computation, 224, 848-865.

[16] Guckenheimer, J., \& Holmes, P. J. (1983). Nonlinear Oscillations, Dynamical Systems, and Bifurcations of Vector Fields. Springer, New York.

[17] Wiggins, S. (2003). Introduction to Applied Nonlinear Dynamical Systems and Chaos. Springer, New York.

[18] Kuznetsov, Y. (2004). Elements of Applied Bifurcation Theory. Springer, New York.

[19] Zhu, L., \& Zhao, M. (2009). Dynamic complexity of a host-parasitoid ecological model with the Hassell growth function for the host. Chaos, Solitons \& Fractals, 39(3), 1259-1269.

[20] Din, Q., Gumus, O. A., \& Khalil, H. (2017). Neimark-Sacker bifurcation and chaotic behaviour of a modified host-parasitoid model. Zeitschrift fur Naturforschung A, 72(1), 25-37. 